\title{
De caminatas a los juzgados: Análisis del discurso de los medios de prensa sobre el proyecto minero Crucitas
}

\author{
José Andrés Díaz González \\ Instituto de Estudios Sociales en Población. Universidad Nacional, Heredia, Costa Rica. \\ Email: joseandres.diaz@ucr.ac.cr
}

Resumen: El presente artículo analiza cuál es la imagen presentada en el contenido de cuatro medios de prensa escrita: La Nación, Diario Extra, el Semanario Universidad y El País, acerca del conflicto en torno al proyecto minero Crucitas; con el propósito de comprender cuál es el discurso que busca transmitir cada medio sobre el tema, especialmente, en procura de observar la manera en que interpretan este conflicto social. El periodo de estudio seleccionado abarca del 8 de mayo del 2010 al 7 de mayo del 2011, es decir, el primer año de la administración Chinchilla Miranda. ${ }^{1}$

Palabras clave: Conflictos Ambientales, Medios de Comunicación, Movimientos Sociales, Análisis de Discurso, Costa Rica.

\section{Walking to the courts: Discourse analysis of the media about the mining project Crucitas}

\begin{abstract}
The paper discusses the image presented in the contents of four newspapers: La Nación, Diario Extra, Semanario Universidad and El País, about the conflict over the mining project Crucitas, seeking to understand the discourse that each newspaper seeks to express about the issue, especially the way they interpret this social conflict. The selected study period runs between May 8, 2010 and May 7, 2011, the first year of the Chinchilla Miranda's Administration.

Key words: Environmental Conflicts, Mass Media, Social Movements, Discourse Analysis, Costa Rica.

\section{Caminhando para os tribunais: análise do discurso da mídia sobre o projeto de mineração Crucitas}

Resumo: Este artigo discute o que a imagem apresentada no conteúdo de quatro suportes de impressão: La Nación, Diario Extra, el Semanario Universidad, El País, sobre o conflito em torno do projeto de mineração Crucitas, com o objetivo de entender o que é o discurso que procura transmitir cada mídia sobre o assunto, especialmente na tentativa de observar a maneira como eles interpretam esse conflito social. O período de estudo selecionado é executado a partir de 08 de maio de 2010 a 7 de maio de 2011, ou seja, o primeiro ano da administração Chinchilla Miranda.

Palavras-chave: Conflitos Ambientais, Mídia, Movimentos Sociais, Análise do Discurso, Costa Rica. 
"Viva la naturaleza, no a la minería”, gritaron ecologistas presentes en el juzgado al momento de conocerse el fallo.

(El País, 24/11/2010)

El presente artículo analiza cuál es la imagen presentada en el contenido de cuatro medios de prensa escrita: La Nación (LN), Diario Extra (DE), el Semanario Universidad (SU) y El País (EP); acerca del conflicto en torno al proyecto minero Crucitas; con el propósito de comprender cuál es el discurso que busca transmitir cada medio sobre el tema, especialmente, en procura de observar la manera en que interpretan este conflicto social.

El periodo de estudio seleccionado abarca del 8 de mayo del 2010 al 7 de mayo del 2011, es decir, el primer año de la administración Chinchilla Miranda; el cual abarca el periodo en el que el conflicto trata de ser solucionado en los tribunales, y permite observar la lectura que hacen los medios acerca de las acciones de la administración Chinchilla Miranda en torno al conflicto generado por el posible desarrollo de un proyecto minero a cielo abierto en la comunidad de Crucitas

\section{Breve reseña sobre el conflicto Crucitas}

Crucitas $^{2}$ es como se le denomina a un proyecto minero, localizado en la comunidad de Las Crucitas de Cutris, cantón de San Carlos (Alajuela, Costa Rica); el cual pretendía la extracción de oro por medio de minería a cielo abierto por parte de la empresa Industrias Infinito S.A., subsidiaria de la transnacional canadiense Infinito Gold S.A. El descontento y la protesta social se generan a partir de la concesión otorgada en el 2001 por el Gobierno de Costa Rica a esta empresa para explotar minerales, principalmente oro, por un período de 10 años, en 305.9 hectáreas (Resolución N $^{\mathrm{O}}$ R-578-2001-MINAE).

Durante la administración de Abel Pacheco de la Espriella (20022006), este proyecto minero no logra desarrollarse, ya que dicha administración no favorece la realización de esta actividad y, más bien, mediante el decreto $\mathrm{N}^{\circ}$ 30477-MINAE se declara una moratoria a la realización de este tipo de actividades mineras.

Durante la segunda administración de Oscar Arias Sánchez (20062010) se anula el decreto de moratoria a la actividad minera firmado por Pacheco de la Espriella. Además, se brinda un abierto apoyo al desarrollo del proyecto minero en Crucitas, ya que se firma el decreto $\mathrm{N}^{\circ} 34801$ MINAET, el cual declara como de interés público y conveniencia nacional al proyecto, lo cual permite a la empresa Industrias Infinito el desarrollo de las obras de infraestructura en áreas de protección de quebradas y ríos, así como la tala de árboles, incluyendo especies en veda como el almendro amarillo. 
En ese contexto los sectores ambientalistas se pronuncian en contra del manejo que se le da a Crucitas por parte del gobierno, emprendiendo protestas y distintas acciones legales, por ejemplo la presentación de ocho recursos de amparo ante la Sala Constitucional, solicitando la declaratoria de inconstitucionalidad de los permisos y decretos ejecutivos firmados en beneficio del proyecto minero Crucitas; los cuales fueron todos rechazados por este ente en abril del 2009, dándole permiso a la empresa Industrias Infinito para que continuara con el desarrollo del proyecto minero (CRH/ EFE, CRH: 16/4/2009).

Los grupos opositores a Crucitas también realizan algunas acciones de protestas. Entre las acciones de protesta se destacan dos caminatas, las cuales parten desde Casa Presidencial hasta el Proyecto Crucitas, con un recorrido de 180 kilómetros; y una huelga de hambre realizada por manifestantes en frente de Casa Presidencial, con el objetivo de solicitarle a la Presidenta de la República, Laura Chinchilla Miranda que tomara acciones para detener la minería en Crucitas.

Finalmente, tras la sentencia dictada por el Tribunal de lo Contencioso Administrativo de Costa Rica, el 24 de noviembre de 2010, se anula la concesión minera en Crucitas a la empresa canadiense y le obliga a indemnizar al Estado de Costa Rica por los daños ambientales ocasionados.

\section{Medios, discurso hegemónico y construcción de la realidad social}

A inicios del siglo XXI es casi indiscutible pensar que los medios de comunicación juegan un papel fundamental en la construcción de la opinión pública y, de esta manera, contribuyen en la construcción de los imaginarios sociales a cerca de diferentes temáticas que captan el interés de la sociedad. Por lo tanto, los estudios sobre los medios de comunicación de masas se basan en la premisa de que estos crean efectos sobre la sociedad; sin embargo, el impacto y grado de incidencia que tienen los medios realmente sobre la sociedad es uno de los puntos más discutidos desde las distintas perspectivas de las teoría de la comunicación de masas (McQuail, 1996: 319).

Por tal motivo, el presente artículo trata de determinar cuál es la imagen sobre los acontecimientos relacionados con el proyecto minero Crucitas manifestada por los medios monitoreados. La importancia de determinar la imagen proyectada por los medios se debe a que se parte de la idea de que ellos presentan o construyen el contenido de sus noticias, para que estas respondan a su propia lógica de interpretación (discurso) de los acontecimientos que reseñan:

El contenido de los medios se puede basar en lo que sucede en el mundo material aunque separa y sólo destaca ciertos elementos, y la 
propia estructura lógica del medio se impone sobre dichos elementos. La realidad es manipulada necesariamente cuando los sucesos y las personas se vuelven a colocar dentro de noticias transmitidas... Los medios pueden imponer su propia lógica al ensamblar materiales en una serie de maneras, incluyéndose el énfasis de ciertos comportamientos y personas y estereotiparlas... Una de las maneras más obvias en que el contenido de los medios estructura un ambiente simbólico es poniéndole una mayor atención (en la forma de más tiempo, mayor importancia y demás) a ciertos sucesos, personas, grupos y lugares que a otros. (Shoemaker y Reese, 1994: 38)

No hay que olvidar además que las noticias, como una unidad de información que transmite el medio de comunicación no solamente se encuentra definida por el ente emisor, sino también por el receptor que tiene interés o encuentra cierto valor de informarse sobre una serie de acontecimientos determinados (McQuail, 1996: 372). Por lo tanto, el seguimiento que realizan los periódicos sobre el tema de Crucitas puede también interpretarse como la existencia en un interés de la población costarricense sobre este. Además, no hay que obviar el hecho que si bien los medios de comunicación no le indican a la población cómo pensar, sí pueden influir indicándoles en qué pensar (Bustos Mora, 2011: 111), en otras palabras, los medios pueden desarrollar una agenda propia con el propósito de colocar la atención de la opinión pública sobre determinados temas, al mismo tiempo que se ignoran otros.

A través de la realización de un análisis de contenido de las noticias publicadas periodo de estudio indicado por La Nación, Diario Extra, el Semanario Universitario y El País, se busca esbozar cuál es el discurso sostenido por dichos medios respecto al conflicto social que se gestaba por la posible realización de un proyecto de minería a cielo abierto en la comunidad de Crucitas y las acciones y posiciones tomadas por diferentes actores sociales (Gobierno, grupos ecologistas, empresa privada, etc.) que intervinieron en este. En cuanto al discurso, este se puede entender como una práctica social [] una forma de acción entre la personas que se articulan a partir del uso lingüístico contextualizado, ya sea oral o escrito (Casimiglia y Tusón, 1999: 15. El resaltado es original). Por su parte, Teun van Dijk al referirse a discurso como práctica social manifiesta que: [1]a utilización discursiva del lenguaje no consiste solamente en una serie ordenada de palabras, cláusulas, oraciones y proposiciones, sino también en secuencias de actos mutuamente relacionados. (van Dijk, 2000: 21)

Asimismo, existe una relación directa entre discurso y dominación/ hegemonía, ya que: los discursos de un grupo de poder pueden ser tales que otros producirán las intenciones y realizarán los actos como si no hubiese ninguna coacción y estos fueran consistentes con sus propios deseos e intereses (van Dijk, 2000: 43). De esta manera, los distintos grupos intentan, por medio de discurso, ejercer su poder y establecerse como grupo domi- 
nante o hegemónico; sin embargo, el uso del discurso como mecanismo de dominación no es explícito sino implícito, lo cual dificulta su percepción y facilita la posibilidad de que el grupo dominante logre manipular a los dominados, de manera que su ideología vaya siendo asimilada, estableciéndose como natural y logrando así la hegemonía. (Murillo y Vergara, 2004: 209)

Sobre la hegemonía, Gramsci señala que esta encierra y presupone unidad intelectual y ética [además de económica y política] conforme a una concepción de lo real que ha superado al sentido común (citado por Murillo y Vergara, 2004: p. 208); es decir, la hegemonía supone que el grupo dominante ha ejercido de tal manera su Poder que los otros sectores han asimilado como suya la ideología del grupo dominante y actúan inconscientemente de acuerdo a ella.

Los medios de comunicación se convierten en mecanismos para la construcción de la hegemonía, ya que al servir como medio de difusión masiva de ideas inciden directamente en la formación de la conciencia político-social de la población, brindándole elementos para la interpretación de los acontecimientos o coyunturas a la que se enfrenta para que esta sea o trate de ser- congruente con la visión de los grupos dominantes, o que controlan dichos medios de comunicación (Esteinou Madrid, 1992: 42-48 ). Por lo tanto, la imagen que proyecta los contenidos de los medios estudiados sobre el caso de Crucitas, busca brindar a la ciudadanía elementos para que interprete los acontecimientos en torno a este de una manera determinada; sino que busca asimismo transmitir elementos a la población para reforzar en esta una determinada visión de cómo observar e interpretar los acontecimientos sociales en general.

\section{Metodología}

Para efectos del presente artículo, se define periódico o prensa escrita como un medio de comunicación (escrita) dirigido hacia la masa o un público amplio, es decir, de carácter público o abierto, caracterizado por su aparición regular, la base comercial y su finalidad de informar sobre múltiples tópicos o temas (McQuail, 1996: 29); de esta manera el carácter impreso del medio no es un factor determinante para definirlo como periódico.

La anterior definición se ha establecido debido a que se trabaja al mismo tiempo con periódicos que cuentan tanto con una edición impresa como digital (La Nación, Diario Extra y el Semanario Universidad), así como periódicos que solo funcionan como medios digitales (El País). La anterior decisión obedece a dos razones, la primera, de carácter operativo, ya que el acceso a la edición digital de los medios facilita el proceso de recolección de información con el propósito de realizar el análisis de contenido de dichos textos. 
Tabla 1. Categorías utilizadas para el análisis de contenido de las noticias publicadas acerca de la minería en Crucitas por La Nación, Diario Extra, El Semanario Universidad y EI País. (del 8 de mayo del 2010 al 7 de mayo del 2011)

\section{Categoría}

Lugares

Actores

Proceso Judicial

Acciones de protesta

\section{Descripción}

A Referencia a ubicaciones espaciales identificadas por el medio en el que ocurren hechos relacionados con el tema de la minería en Crucitas.

1. Espacios que podrían recibir un determinado impacto por el desarrollo del proyecto minero en Crucitas.

A. Actores que cada medio señala que participa durante los conflictos en torno al tema de Crucitas.

1. La posición de los actores respecto a Crucitas

A Acciones legales interpuestas para detener el proyecto minero en Crucitas

A Referencias al Juicio Contencioso Administrativo realizado para determinar si la concesión dada a Industrias Infinito cumplía con todos los requisitos legales.

A Acciones y estrategias que grupos contrarios al proyecto minero en Crucitas realizan en procura de detener la realización de este.

Fuente: Construcción propia. Noviembre, 2011.

La segunda razón es corresponde al auge que están teniendo los medios digitales como pieza clave en el desarrollo de la llamada sociedad de la información. El auge del internet ha llevado a muchos medios de comunicación escrita impresos a desarrollar versiones digitales, con el propósito tanto de mejorar su imagen, como para posicionar su contenido en esta plataforma de comunicación (Meso Ayerdi, 2008: 61-62).

Se realizó un análisis de contenido de la información recopilada, con el propósito de identificar así las categorías utilizadas por los medios para presentar la información contenida en las noticias publicadas por ellos. Para la realización del análisis de contenido se recurrió a la metodología de análisis propuesta por Kaus Krippendorff (1997), de esta forma, partiendo de la palabra como unidad básica de análisis se procedió a realizar un conteo de frecuencias, ${ }^{3}$ el cual es la forma más utilizada para representar y analizar textos utilizando el análisis de contenido (Krippendorff, 1997: 162). El conteo de frecuencias permite identificar las palabras más utilizadas en el contenido de los textos, así como facilita observar su importancia relativa respecto a las otras palabras presentes en el texto.

Posteriormente, se recurre a lo que Krippendorff (1997: 168) denomina como análisis de contingencia, el cual consiste en inferir las asociaciones presentes en las unidades de análisis seleccionadas (en este caso las palabras) a partir de los símbolos que estas buscan representar en el mensaje, es decir, se procedió a la construcción de conglomerados de palabras, las cuales buscaban representar un elemento o símbolos específicos (tópicos), y al mismo tiempo estas se agruparon en categorías, las cuales permi- 
ten observar cuáles son los principales elementos incluidos en el contenido de las noticias sobre Crucitas y que elementos lo componen, asimismo, facilita identificar las semejanzas y diferencias en el contenido de los diversos periódicos estudiados..

En total se identificaron cuatro categorías: Lugares, Actores, Proceso Judicial y Acciones de protesta. Debe señalarse que necesariamente no todas las categorías están presentes en todos los medios estudiados, o no tienen la misma relevancia en su contenido; lo anterior es importante para tratar de visualizar las diferencias en el discurso sostenido por cada medio en torno al tema de Crucitas.

Para realizar el presente análisis se recopilaron las noticias publicadas por La Nación, Diario Extra, El País y el Semanario Universidad, entre el 8 de mayo del 2010 y el 7 de mayo del 2011. En total se recolectaron 336 noticias, la mayor cantidad fue publicada por el medio digital El País (212 noticias), seguido por La Nación (63 noticias), Diario Extra (43 noticias) y el Semanario Universidad (18 noticias). ${ }^{4}$

Debe indicarse que la información recopilada corresponde únicamente a noticias y no incluye los editoriales ni los artículos de opinión publicados sobre el tema de Crucitas durante ese periodo por dichos medios relacionados con el tema de Crucitas. Esto debido a que lo que se busca es observar cómo cada medio presenta la información referente a este caso, por lo que utilizar los artículos de opinión publicados en los medios provocaría que se analizara el contenido de posiciones que no necesariamente correspondan a la visión del medio.

\section{Análisis de contenido de las noticias sobre Crucitas}

En este apartado se encuentran los resultados del análisis de contenido de las noticias publicadas por La Nación, Diario Extra, el Semanario Universidad y El País, sobre Crucitas. Para efectos de orden, se presentan los resultados a partir de cada una de las categorías encontradas en el contenido de estos medios de prensa escrita, anteriormente señalados: Lugares, Actores, Proceso Judicial y Acciones de protesta.

\section{Lugares}

En cuanto a la primera categoría, Lugares, esta se compone de dos elementos, las referencias a espacio Local y Nacional (ver Cuadro 1). En general, las referencias al espacio Local van ligadas a identificar el lugar donde se llevaría a cabo el proyecto minero, así como el impacto que tendría para la zona y sus alrededores.

Además, las referencias al espacio Local en Diario Extra permite observar la existencia de pugna entre dos posiciones, la primera, basada en la idea que la minería a cielo abierto traería progreso y mejoras en la condi- 
ción de vida de la zona, por ejemplo, se destaca que la empresa Industrias Infinito expresa haber invertido \$23 millones para mejorar las condiciones de vida de las personas de la comunidad por medio de la construcción de caminos y obras similares (Cordero G., DE: 17/11/2010). La segunda posición, es de aquellos que se manifiestan en contra de la realización del proyecto debido a su impacto negativo en el ambiente, en contraste de los beneficios económicos y sociales que supuestamente acarrearía la minería, ese es el caso del Consejo Municipal de San Carlos, el cual aprobó una moción para pedirle al Gobierno la anulación de los permisos del Proyecto Minero Crucitas (Artavia, DE: 29/5/2010).

Cuadro 1. Tópicos que conforman la categoría "Lugares", en el contenido de las noticias relacionadas a Crucitas. Del 8 de mayo del 2010 al 7 de mayo del 2011. (Por medio de comunicación)

\begin{tabular}{|c|c|c|c|c|c|c|c|c|}
\hline \multirow{2}{*}{ Tópicos } & \multirow{2}{*}{$\begin{array}{c}\text { El País } \\
\text { Palabras }\end{array}$} & \multicolumn{2}{|c|}{ Diario Extra La Nación } & \multicolumn{5}{|c|}{ Semanario Universidad } \\
\hline & & Frecuencia & Palabras & Frecuencia & Palabras & Frecuencia & Palabras & Frecuencia \\
\hline \multirow{3}{*}{ Local } & Crucitas & 605 & Crucitas & 174 & Crucitas & 153 & Crucitas & 92 \\
\hline & San Carlos & 196 & San Carlos & 63 & San Carlos & 72 & & \\
\hline & Zona Norte & 156 & Cutris & 40 & Cutris & 45 & & \\
\hline \multirow{3}{*}{ Nacional } & País & 221 & País & 49 & País & 42 & Pais & 48 \\
\hline & Nacional & 345 & Nacional & 48 & Nacional & 43 & Nacional & 41 \\
\hline & Costa Rica & 366 & Costa Rica & 25 & & & Costa Rica & 28 \\
\hline Total & & 1889 & & 399 & & 355 & & 117 \\
\hline
\end{tabular}

En cuanto a La Nación, el Semanario Universidad y El País, las referencias al espacio Local son, principalmente, para ubicar el sitio donde se desarrollaría la actividad minera en cuestión. Sin embargo, El País hace un especial énfasis al impacto negativo que tendría la minería sobre ésta (Elpais.cr, EP: 11/07/2010)

En cuanto el espacio Nacional, Diario Extra mantiene una lógica similar con lo expuesto en el espacio Local, por un lado, se presentan los beneficios que el país en su conjunto podría obtener de realizarse el proyecto, como por ejemplo: los impuestos que generaría la actividad minera (Artavia, DE: 15/6/2010), el bajo impacto ambiental que acarrearía la minería(Cordero G., DE: 17/11/2010) y como el proyecto habría -supuestamente- cumplido con la legislación y procedimientos establecidos en Costa Rica para su aprobación (Cordero G, DE: 12/10/ 2010). Del lado contrario, se encuentra la información que manifiesta cómo la mayoría de la población costarricense se encuentra opuesta al desarrollo del proyecto minero en Crucitas (Artavia, DE: 2/7/2010) o cómo el país se pone a la vanguardia en la región en la protección al medio ambiente al prohibir la minería a cielo abierto (Cordero G., DE: 11/2/2011).

Por su parte, las referencias al plano Nacional utilizadas por La Nación, van enfocadas en mostrar las repercusiones positivas o negativas 
que tendría Costa Rica de aprobarse o rechazarse la explotación minera en Crucitas (Álvaro, LN: 26/7/2010 y Oviedo, LN: 27/7/2010), así como las obligaciones en materia de legislación ambiental y tratados internacionales que el país se compromete a cumplir y por los cuales no podría llevarse a cabo dicho proyecto minero (Mata, LN: 2/8/2010 y Loaiza, LN: 16/08/2010).

El contenido del Semanario Universidad y El País relacionado con lo Nacional se enfocan con el posible impacto negativo que puede traer el desarrollo del proyecto minero en Crucitas, así como la minería en general, para Costa Rica (Córdoba, SU: 16/6/2010 y Elpais.cr, EP: 3/08/2010). En el caso de El País lo Nacional se utiliza también como una referencia para contraponerse a la Extranjero, el cual se identifica como el lugar del que provienen intereses contrarios a los del país, y que buscan dañar o perjudicar el bienestar de la sociedad costarricense (Salazar, EP: 10/05/2010).

\section{Actores}

En cuanto la categoría actores, si bien cada medio revisado identifica una cantidad mayor o menor de actores que intervienen activamente en el conflicto generado por Crucitas (ver cuadro 2), es posible agruparlos en dos grupos: promotores o defensores del proyecto minero y opositores a este.

En cuanto a los promotores de Crucitas, todos los medios revisados coinciden que Industrias Infinito es la principal promotora del proyecto, la cual busca desmentir o minimizar los posibles impactos ambientales que generaría la extracción de oro por medio la minería a cielo abierto, a la vez que defiende la legalidad de lo actuado para el otorgamiento de la concesión minera en Crucitas y pone énfasis en los beneficios que supuestamente traería la minería a la zona (Córdoba, SU: 20/06/10 y Cordero G., DE: 17/11/2010). El Semanario Universidad reporta que esta empresa es denunciada por tratar de influir en la campaña electoral municipal, del Cantón de San Carlos, de diciembre del 2010, con el propósito de perjudicar a aquellos grupos políticos que se oponían al desarrollo del proyecto minero en Crucitas (Córdoba, SU: 16/11/2010). Por su parte, El País recalca casi constantemente que se trata de una empresa extranjera:

En Costa Rica, sin hacer acusaciones, despierta sospecha el gran interés de los titulares del Gobierno anterior con la emisión de un expedito decreto para permitir la minería de oro a cielo abierto, en defensa del proyecto minero Las Crucitas, propiedad de la transnacional canadiense Infinito Gold. (Salazar, EP: 10/05/2010)

Parece que uno de los objetivos de El País es dejar claro que Industrias Infinito es una empresa extranjera, lo cual sugiere que es utilizado para reforzar dos posturas: la primera, la intervención de un ac- 
tor externo (no nacional) en las decisiones adoptadas por distintos entes del Gobierno costarricense; y la segunda, que al tratarse de una empresa extranjera, el bienestar del país y sus habitantes no son su prioridad, por lo que están dispuestos a realizar acciones, como campañas publicitarias, para ganar el favor de la opinión pública a pesar de las posibles consecuencias negativas que tendría el desarrollo de la minería (Elpais.cr, EP: 3/06/2010)

El siguiente actor identificado por El País, La Nación y el Semanario Universidad como principal promotor de Crucitas es Oscar Arias Sánchez, respecto del cual se destaca que durante su segunda administración se firma el decreto que declara ese proyecto minero como de interés público (Murillo, LN: 15/7/2010 y Murillo, LN: 23/7/2010; Chacón, SU: 7/12/2010; y Salazar, EP: 13/10/2010); incluso cómo continúa apoyando el proyecto luego de dejar la Presidencia de la República (Elpais.cr, EP: 8/05/2010; Elpais.cr, EP: 25/09/2010 y EFE, EP: 26/ $11 / 2010)$.

El Semanario Universidad y El País, además, cuestionan si lo actuado por Oscar Arias Sánchez respecto Crucitas podría contener violaciones a la ley (Chacón, SU: 7/12/2010); especialmente tras el fallo de Tribunal Contencioso Administrativo que declara ilegal la concesión otorgada a Industrias Infinito (Elpais.cr, EP: 26/11/2010; Elpais.cr, EP:15/12/2010 y Elpais.cr, EP: 19/12/2010).

Además, El País y el Semanario Universidad cuestionan los vínculos que mantenía Oscar Arias con Industrias Infinito, tanto por medio de donaciones realizadas por esta empresa a la Fundación Arias, una organización creada por el propio Arias Sánchez a finales de la década de 1980 (Elpais.cr, EP: 28/04/2011 y Elpais.cr, EP: 29/04/2011), como por su relación cercana con personas estrechamente relacionadas con esta empresa (Córdoba, SU: 30/6/2010)

La Nación y el Semanario también identifican a Rodrigo Arias, hermano de Oscar Arias y Ministro de la Presidencia durante las dos administraciones de este, como un actor en defensa de Crucitas; especialmente se le identifica con críticas al fallo judicial que declara ilegal el proceso de concesión de la minería en Crucitas, así como a la actuación del Gobierno por no defender con más fuerza dicho proyecto (Esquivel, LN: 25/11/2010 y Esquivel y Agüero, LN: 25/11/2010). 


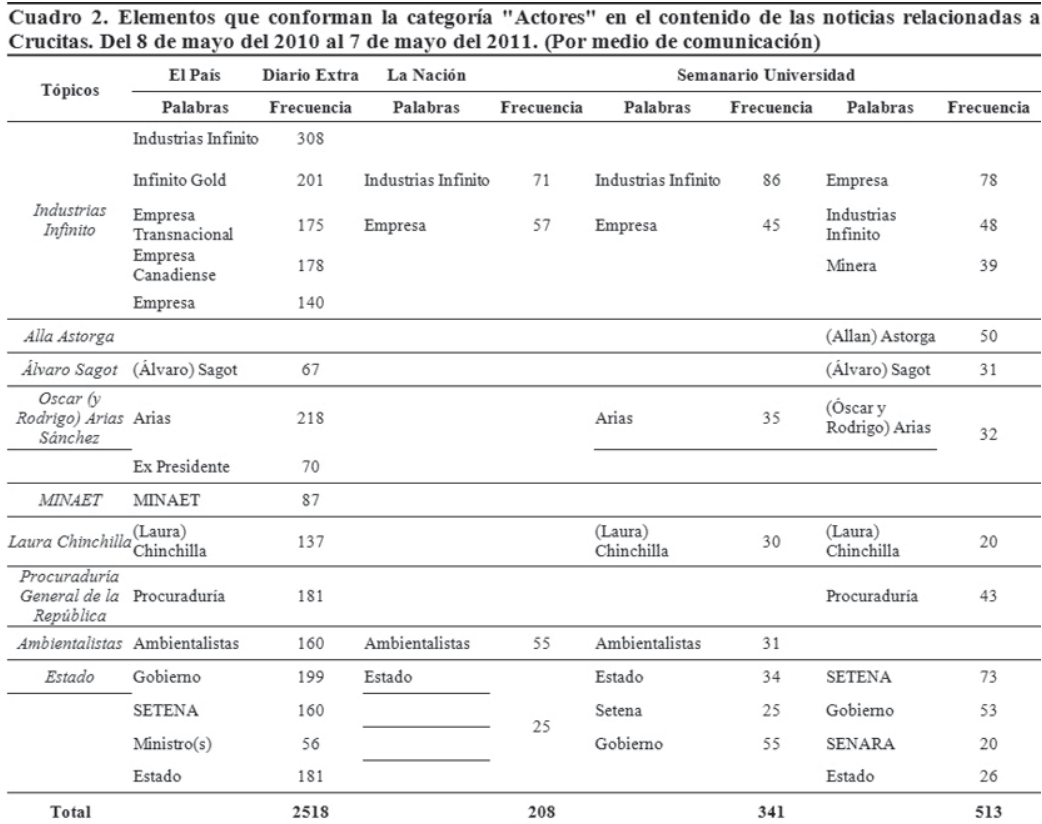

Fuente: Construcción propia, a partir de la información encontrada en Diario Extra, el Semanario Universidad, El Pais y La Nación, del 8 de mayo de 2010 al 7 de mayo de 2011. Noviembre, 2011.

En cuanto al Estado como actor, el Semanario Universidad mira de manera crítica las acciones realizadas por las diferentes instituciones públicas involucradas en el proceso de concesión de la minería en Crucitas (Córdoba, SU: 12/1/2011), así como en el desarrollo del juicio contencioso administrativo que se llevó a cabo para definir la legalidad de esta (Córdoba, SU: 25/1/ 2011 y Córdoba, SU: 23/2/2011), esto a pesar que el desarrollo del proyecto minero Crucitas correspondía a una política del Gobierno y no del Estado..

Por su parte, El País y Diario Extra identifican que el Estado (o Gobierno) juega un doble papel, ya que defiende tanto su actuación en el otorgamiento de los permisos para el funcionamiento de la mina a cielo abierto en Crucitas, así como al propio proyecto; y al mismo tiempo, se muestran los esfuerzos del Gobierno para prohibir (primero por decreto y luego mediante una ley) la minería a cielo abierto en el país (Elpais.cr, EP: 30/07/2010) e impulsar la imagen de Costa Rica como país verde (Elpais.cr, EP: 28//07/2010 y Elpais.cr, EP: 29/07/2010.

Además, El País expresa en varias notas periodísticas que los entes encargados de los permisos ambientales, como SETENA, ignoran advertencias realizadas por diferentes actores sociales acerca del peligro que representa la minería en Crucitas (Elpais.cr, EP: 13/05/2010 y Elpais.cr, EP: 14/05/2010). 


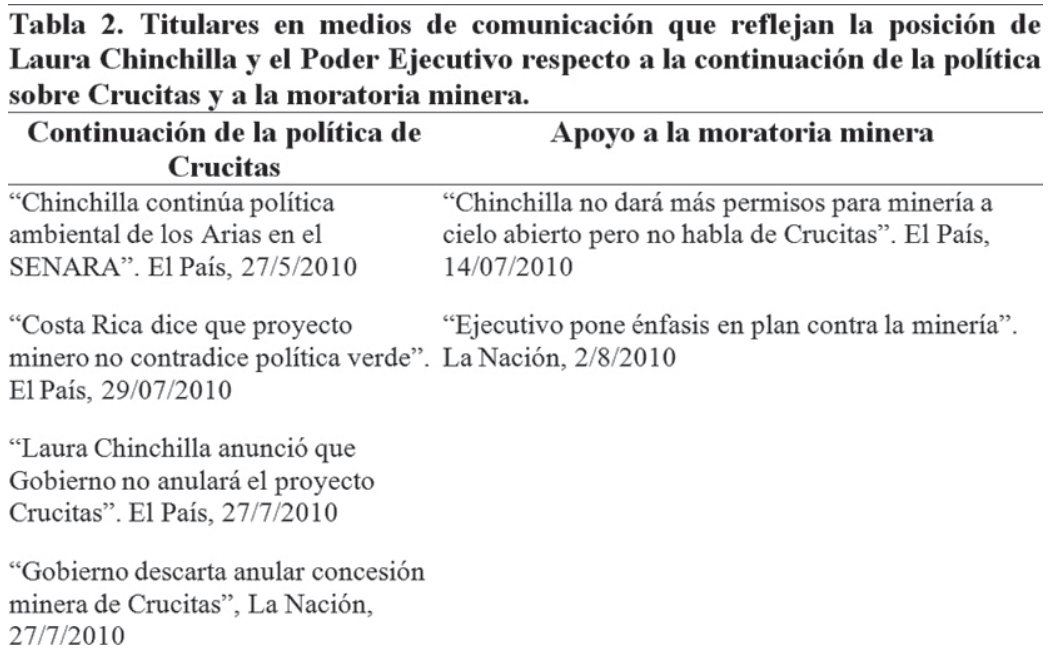

Fuente: Construcción propia, a partir de la información recolectada en los periódicos La Nación y El País, entre el 8 de mayo del 2010 y el 7 de mayo del 2011. Marzo, 2012.

En cuanto a la actuación de la Presidenta de la República, Laura Chinchilla Miranda, en la información reportada tanto por El País, La Nación y el Semanario Universidad, se muestra que ella mantiene una doble posición sobre el tema de la minería en Costa Rica; ya que si bien por un lado manifiesta estar en contra del desarrollo de nuevos proyectos de minería a cielo abierto en Costa Rica (Elpais.cr, EP: 14/07/2010; Mata, LN: 2/08/2010 y Córdoba, SU: 2/06/2010), por otro lado continúa, como se indicó, con la política de Arias Sánchez sobre el tema de Crucitas (Elpais.cr, EP: 9/05/2010 y Elpais.cr, EP: 27/5/2010) y no realiza ninguna acción en procura de detener el desarrollo de dicho proyecto o no toma una postura clara sobre este (Elpais.cr, EP: 15/10/2010; Elpais.cr, EP: 23/07/2010; Elpais.cr, EP: 27/07/2010; Murillo, LN: 15/ 7/2010 y Ramírez, SU: 23/06/2010). Ejemplo de esta doble posición puede observarse en los titulares de algunos de los medios de comunicación consultada (ver Tabla 2).

Como un último actor en favor de la minería en Crucitas, El País identifica a la Procuraduría General de la República (PGR). El País presenta a la PGR como el ente que utiliza el Poder Ejecutivo para dar apoyo a Industrias Infinito, así como para defender lo actuado por el propio Gobierno en el juicio contencioso administrativo seguido para determinar la legalidad de dicha concesión. Es importante señalar que otros actores pertenecientes al Gobierno, como la Asamblea Legislativa, así como otros actores sociales critican las acciones de la PGR en 
defensa de Crucitas (Elpais.cr, EP: 31/10/2010; Elpais.cr, EP: 21/01/ 2011 y Elpais.cr, EP: 28/01/2011); asimismo, se cuestiona la intervención de Oscar Arias Sánchez, en las acciones de la PGR para apelar la sentencia dada por el Tribunal Contencioso Administrativo que anuló la concesión minera de Crucitas (Elpais.cr, EP: 2/11/2010; Elpais.cr, EP: 19/01/2011 y Elpais.cr, EP: 31/01/2011)

En cuanto a los actores que se manifiestan en contra de Crucitas, primero, se encuentran los grupos ambientalistas. Al respecto, tanto Diario Extra, La Nación y El País presentan a este como un ente amorfo, como un conjunto o movimiento de personas sin identificación especial de los distintos grupos y posturas que pueden pertenecer a este movimiento ambientalista; aunque si destaca una serie de acciones realizadas por estas personas en procura de mostrar su oposición a la minería en Crucitas (Fuentes, LN: 26/10/2010; Artavia, DE: 14/5/2010; Elpais.cr, EP: 12/07/2010; Cordero, DE: 16/07/2010 y Artavia, DE: 15/ 06/2010).

En cuanto al contenido del Semanario Universidad, es llamativo que en la categoría de Actores no aparecen los ambientalistas o grupos ambientalistas como actores importante en los acontecimientos reportados por dicho medio (ver cuadro 2). En contraste, este medio, al igual que El País y, en menor medida, Diario Extra, identifican como actor relevante en el grupo de oposición a Crucitas a Álvaro Sagot, abogado ambientalista que no sólo manifiesta su oposición al proyecto en diferentes foros y debates (Artavia, DE: 14/5/2010, Elpais.cr, EP: 11/05/ 2010 y Elpais.cr, EP: 23/06/2010), sino también es uno de los abogados que llevan el juicio contra Industrias Infinito.

También El País y el Semanario Universidad identifican en su contenido como actor importante a Allan Astorga, Ex-Secretario General de SETENA y Profesor de la Escuela de Geología de la Universidad de Costa Rica, y quién fuera una de las principales figuras académicas en contra del proyecto minero en Crucitas (Córdoba, SU: 1/12/2010 y Elpais.cr, EP: 23/06/2010)

Se aprecia, entonces, como estos medios parecen replicar la tendencia de personalizar y masculinizar la protesta y lucha social que se ha venido presentando en Costa Rica en otras situaciones anteriores de conflicto social (Mora y García, 2008. 103), no solo rescatando el papel de los actores con conocimiento técnico, sino presentado cómo fueron estos los que al final consiguieron detener la realización del proyecto Crucitas (Elpais.cr, EP: 23/10/2010; Elpais.cr, EP: 25/11/2010 y Elpais.cr, EP: 13/11/2010), algo que la protesta social pareciera que fue incapaz de hacer (Elpais.cr, EP: 2/11/2010). 
Cuadro 3. Tópicos que conforman la categoria "Proceso Judicial" en el contenido de las noticias relacionadas a Crucitas. Del 8 de mayo del 2010 al 7 de mayo del 2011. (Por medio de comunicación)

\begin{tabular}{|c|c|c|c|c|c|c|c|c|}
\hline \multirow{2}{*}{ Tópicos } & \multicolumn{2}{|c|}{ El Pais } & \multicolumn{2}{|c|}{ Diario Extra } & \multicolumn{2}{|c|}{ La Nación } & \multicolumn{2}{|c|}{ Semanario Universidad } \\
\hline & Palabras & Frecuencia & Palabras & Frecuencia & Palabras & Frecuencia & Palabras & Frecuencia \\
\hline \multirow{10}{*}{$\begin{array}{c}\text { Juicio } \\
\text { contencioso } \\
\text { administrativo }\end{array}$} & Tribunal & 309 & Tribunal & 66 & Tribunal & 87 & Tribunal & 49 \\
\hline & $\begin{array}{l}\text { Contencioso } \\
\text { Administrativo }\end{array}$ & 178 & $\begin{array}{l}\text { Contencioso } \\
\text { Administrativo }\end{array}$ & 45 & $\begin{array}{l}\text { Contencioso } \\
\text { Administrativo }\end{array}$ & 44 & $\begin{array}{l}\text { Contencioso } \\
\text { Administrativo }\end{array}$ & 42 \\
\hline & Juicio & 136 & Juicio & 44 & Juicio & 48 & Juicio & 25 \\
\hline & Caso & 125 & Caso & 43 & Caso & 32 & Proceso & 28 \\
\hline & Sentencia & 152 & Fallo & 43 & Fallo & 26 & Abogado & 24 \\
\hline & Proceso & 118 & Proceso & 37 & Proceso & 27 & Sentencia & 38 \\
\hline & & & Jueces & 25 & Sentencia & 47 & & \\
\hline & & & & & Abogado & 33 & & \\
\hline & & & & & Ley & 34 & & \\
\hline & & & & & Jueces & 26 & & \\
\hline Sala & Sala IV & 161 & Sala IV & 65 & Sala IV & 44 & Sala IV & 64 \\
\hline Constitucional & Magistrados & 27 & Constitucional & 25 & & & Recurso & 35 \\
\hline Total & & 1206 & & 393 & & 448 & & 305 \\
\hline
\end{tabular}

Fuente: Construcción propia, a partir de la información encontrada en Diario Extra, el Semanario Universidad, El Pais y La Nación, del 8 de mayo de 2010 al 7 de mayo de 2011. Noviembre, 2011.

Con lo anterior, pareciera que la información presentada en los contenidos de los medios analizados, ha tendido a personalizar o individualizar la participación en contra del proyecto en Crucitas, dando un menor seguimiento a las acciones realizadas por los movimientos sociales o colectivos de personas que se manifestaran en contra de la minería. Esta idea se refuerza al constatar que el contenido de las noticias publicadas por estos medios sobre las acciones de protesta realizadas por diferentes grupos, no tienen la suficiente relevancia para construir una categoría que las contenga.

\section{Proceso Judicial}

La categoría Proceso Judicial es una de las más importantes identificadas en el contenido de las noticias publicadas sobre Crucitas en los medios estudiados; su contenido engloba las acciones legales que se realizaron para tratar de detener el proyecto minero en Crucitas, con especial énfasis en el juicio contencioso administrativo llevado a cabo entre septiembre y noviembre del 2010 (Ver cuadro 3).

En el caso de Diario Extra, el Gobierno y, en especial, el Gabinete y la Presidenta de la República, Laura Chinchilla, no aparecen identificados como actores relevantes en el contenido de dicho medio, y menos aún en lo que se refiere al Proceso Judicial. Diario Extra presenta el conflicto en torno a Crucitas como un juego en el que se enfrentan la empresa Industrias Infinito y los grupos ambientalistas, dejando de lado la participación del Gobierno en este, y presentando a las instancias públicas (los tribunales de justicia) únicamente como el canal adecuado para resolver dicho enfrentamiento (Cordero, DE: 11/08/2010 y Cordero, DE: 20/09/2010).

De esta manera, la imagen que presenta Diario Extra en sus noticias es la de una Costa Rica donde el Estado de Derecho prevalece (Cordero, DE: 15/09/2010), ya que si bien pueden existir divergencias y conflictos 
entre los intereses privados (Industrias Infinito) y públicos (grupos ambientalistas), al final estas son resueltas mediante los canales institucionales adecuados; y donde el Gobierno es simplemente un espectador que no interfiere para beneficiar a ninguna de las partes en conflicto.

El principal elemento en el contenido de las noticias de La Nación es referente al Proceso Judicial y a las acciones llevadas a cabo en el sistema de justicia para definir la legalidad o ilegalidad de la concesión (Vizcaíno, LN: 4/10/2010; Loaiza, LN: 12/10/2010 y Vizcaíno y otros, LN: 24/11/ 2010). Así, se refuerza la tesis acerca de que predominantemente se visualiza que este conflicto sería resuelto por los canales institucionales, disminuyéndole el peso a la presión que diferentes actores sociales ejercían contra la minería en Crucitas. Lo anterior es claro al observar que no hay una categoría de Acciones de Protesta en el contenido de las noticias de La Nación (ver Cuadro 4); y si bien hay algunas noticias publicadas por este medio sobre las manifestaciones, marchas y huelgas realizadas contra el proyecto minero Crucitas, estas son pocas, e incluso algunas van dirigidas a comunicar cómo el Gobierno minimiza o resta importancia a dichas acciones, por lo cual no hay suficiente material para construir dicha categoría en el contenido de ese medio (Loaiza y Murillo, LN: 19/10/2010 y Fuentes, LN: 26/10/2010).

Sobre el contenido presente en la categoría Proceso Judicial, en el caso del Semanario Universidad, también está compuesta por dos elementos. El primero se relaciona con el juicio contencioso administrativo llevado a cabo para determinar la legalidad o ilegalidad del proyecto minero de Crucitas. Como ya se ha comentado, los medios parecen mantener como pieza central de su discurso cómo el conflicto en torno a Crucitas es trasladado a un espacio institucional para su resolución, y el Semanario Universidad no es la excepción; así, este medio presenta cómo el uso de este tipo de mecanismos dio la razón a los grupos opositores de la minería en Crucitas (Córdoba, SU: 1/12/2010)

El segundo se relaciona con el papel jugado por la Sala IV en la revisión de los diferentes recursos de inconstitucionalidad presentados con el propósito de detener la explotación minera en Crucitas. Al respecto, el Semanario Universidad mantiene una posición crítica respecto al rechazo de dicho recurso por parte de la Sala Constitucional, lo cual permitió que se siguiera llevando adelante el proyecto minero (Ramírez, SU: 12/5/2010 y Córdoba, SU: 30/7/2010).

En cuanto al contenido presente en El País en este caso, si se observa la identificación de Actores que realiza este medio, así como la tendencia presentada en los otros medios ya reseñados, no es de extrañar que el Proceso Judicial sea una de las categorías más importantes en el contenido de las noticias publicadas por El País, y el elemento al que se le dio mayor seguimiento en la información publicada por este medio (Elpais.cr, EP: 17/ 09/2010, Elpais.cr, EP: 4/10/2010; Elpais.cr, EP: 5/10/2010; Elpais.cr, EP: 9/10/2010 y Elpais.cr, EP: 29/10/2010), superando por muy amplio mar- 
gen las referencias a las Protestas contra la minería (ver Cuadro 4). A pesar de ello, es importante señalar que El País sí hace un seguimiento de las acciones realizadas por diferentes grupos y personas para manifestar su oposición a Crucitas, algo que no realizan de manera tan constante los otros medios estudiados.

Podría plantearse, como una posible hipótesis para explicar la situación anterior, que dado que el conflicto en torno a la minería en Crucitas es trasladado a una instancia institucional-técnica para su resolución (el Tribunal Contencioso Administrativo), esto provoca que los medios de comunicación se concentren en lo que está pasando en dicho proceso, dejando de lado las manifestaciones y acciones que diferentes actores sociales realizan en las instancias y espacios no formales (huelgas de hambre, protestas, etc.); produciendo al mismo tiempo que los actores colectivos sean sustituidos por actores técnicos; en este caso, los Ambientalistas, como movimiento social, son desplazados del escenario por ambientalistas técnicos, expertos en temas jurídicos y ambientales que pueden participar y generar criterios que se consideran válidos en los espacios de participación formales en los cuales será resuelto este conflicto.

\section{Acciones de protesta}

Como se mencionó anteriormente, los medios presentan como la categoría más relevante de su contenido el Proceso Judicial, y parece que su discurso se va encaminando a mostrar el espacio judicial como lugar legítimo y más adecuado para dirimir este conflicto. Por lo anterior, no es de extrañar que el seguimiento a las acciones de protesta realizada por grupos opositores a la minería en Crucitas fuera marginadas o excluidas del contenido de la mayoría de los medios estudiados (ver Cuadro 4).

Así, en el caso de Diario Extra, la única acción de protesta a la que se le da cierto monitoreo continuo es a las caminatas que un grupo de personas opositoras a la minería realizan desde Casa Presidencial hasta el proyecto minero en Crucitas y viceversa, para mostrar su oposición al proyecto (Cordero, DE: 12/07/2010; Cordero, DE: 16/07/2010 y Cordero, DE: 19/07/2010); mientras que la huelga de hambre que un grupo de ecologistas realizaron frente a Casa Presidencial con el mismo propósito, apenas tuvo mención en este medio (Cordero, DE: 24/11/2010)

Por su parte, como ya se mencionó, La Nación da un mínimo de seguimiento a la huelga de hambre que realizan un grupo de personas frente a Casa Presidencial, con el objeto de presionar a la Presidenta de la República, para que esta tome acciones para frenar el proyecto minero en Crucitas. En cuanto al Semanario Universidad, las acciones de protesta contra Crucitas no son un elemento importante o relevante en su contenido; esto se puede visualizar debido a que durante el periodo analizado solo se encontró una noticia publicada por el Semanario Universidad que se dedicara exclusivamente a acciones realizadas por grupos para protestar en contra del proyecto minero en Crucitas (Núñez, SU: 25/08/2010). 
Cuadro 4. Tópicos que conforman la categoría "Acciones de Protesta" en el contenido de las noticias relacionadas a Crucitas. Del 8 de mayo del 2010 al 7 de mayo del 2011. (Por medio de comunicación)

\begin{tabular}{clccc}
\hline \multirow{2}{*}{ Tópicos } & \multicolumn{2}{c}{ EI País } & \multicolumn{2}{c}{ Diario Extra } \\
\cline { 2 - 5 } & Palabras & Frecuencia & Palabras & Frecuencia \\
\hline \multirow{2}{*}{$\begin{array}{c}\text { Huelga de } \\
\text { Hambre }\end{array}$} & $\begin{array}{l}\text { Huelga de } \\
\text { Hambre }\end{array}$ & 42 & & \\
& Ayuno & 42 & & \\
\hline Caminata & Caminata & 54 & Caminata & 28 \\
\hline Total & & $\mathbf{1 3 8}$ & & $\mathbf{2 8}$ \\
\hline
\end{tabular}

Fuente: Construcción propia, a partir de la información encontrada en Diario Extra y El País, del 8 de mayo de 2010 al 7 de mayo de 2011. Noviembre, 2011.

En el contenido de El País, en especial, se da seguimiento a las caminatas y a la huelga de hambre, realizada por grupos ambientalistas para solicitar al Gobierno y a la Presidenta de la República, que detuvieran el desarrollo del proyecto minero en Crucitas (Elpais.cr, EP: 5/06/2010; Elpais.cr, EP: 5/07/ 2010; Elpais.cr, EP: 12/07/2010; Elpais.cr, EP: 15/07/2010; Elpais.cr, EP: 1/ 10/2010; Elpais.cr, EP: 2/10/2010 y Elpais.cr, EP: 10/10/2010).

Otro punto relacionado con las acciones de protesta que realizan los grupos opositores a Crucitas que señala El País es cómo la Presidenta de la República, Laura Chinchilla Miranda, toma una posición de ignorar las distintas acciones de protesta ya que, como se indicó antes, su postura era dejar que los tribunales resolvieran el conflicto, por lo que ella y el Gobierno lo único que harían sería obedecer la sentencia de los tribunales (Elpais.cr, EP: 15/10/2010).

Por último, un elemento que resulta interesante en esta categoría es que El País toma una postura de denunciar a otros medios de comunicación, específicamente a La Nación, ya que considera que este periódico adopta una postura de criminalizar la protesta social, reduciendo así las capacidades de la población de manifestarse para oponerse o apoyar distintos temas (Elpais.cr, EP: 12/05/2010). Este tipo de denuncias pueden llevar a inferir que El País apoya este tipo de acciones de la población, lo cual permite explicar por qué este medio da un seguimiento más completo de las acciones de protesta realizadas en contra de Crucitas que la de los otros medios estudiados. Así, en la lectura de la realidad que realiza El País, las acciones de protesta que realiza la ciudadanía parecen formar parte importante de su comprensión del funcionamiento de la realidad social.

\section{A modo de conclusión}

En el presente artículo se ha buscado exponer los contenidos prioritarios en las noticias publicadas por cuatro medios de prensa escrita costarri- 
cense en relación al intento de desarrollar un proyecto minero a cielo abierto en la comunidad de Crucitas, así como las acciones realizadas por distintos grupos ambientalistas en procura de evitar que se llevara a cabo dicho proyecto.

De esta manera, lo que se ha realizado es un recuento de los acontecimientos que los medios de información han reportado, así como el énfasis que han hecho respecto de estos; esto ha permitido ir delineando una imagen sobre la forma en que los medios visualizaron dichos acontecimientos y, cómo buscaron trasmitirlos a la opinión pública. Por lo tanto, esta imagen no necesariamente corresponde a la realidad de los eventos producidos alrededor de Crucitas, así tampoco el análisis que se desprende de este pretende ser un análisis de los conflictos, acciones, movimientos sociales, actores y otros elementos que intervienen en esta coyuntura social, sino que lo que se intenta es dar una explicación de la visión que los medios buscan transmitir sobre este caso por medio de su contenido.

La importancia de estudiar el contenido de las noticias transmitidas por los medios de información radica en que estos juegan un papel crucial en el proceso de construcción de los imaginarios sociales. Al respecto, Luhmann establece que los medios buscan proveer en las sociedades el imaginario de la realidad, es decir, de lo que se entiende y acepta por real; además, es claro en indicar que no son una crónica exacta de lo que ha acontecido, sino que la información por ellos suministrada responde a su idea de verdad según sus propias preferencias sociales y escalas de valor. A pesar de lo anterior, el propio Luhmann reconoce que los medios, de una u otra forma describen a la sociedad; así, por ejemplo, el desarrollo de las sociedades capitalistas ha llevado al predominio de los valores mercantiles en el sistema de medios de comunicación (Luhmann, 2007). Luego, se puede partir de la premisa que si bien los medios de comunicación no indican directamente a las personas cómo deben pensar, si influyen directamente en qué pensar (Bustos Mora, 2011: 111.)

De esta manera, siguiendo a Krippendorff (1997), el análisis de contenido realizado a las noticas seleccionadas permite inferir que características e ideas se destacan y cuáles se invisibilizan por parte de los medios en relación al proyecto minero Crucitas, y como estos se relacionan para constituir la imagen que los medios buscan transmitir a la opinión pública. Así, se puede concluir que la imagen general que transmiten los periódicos estudiados sobre el conflicto social surgido en relación al proyecto minero Crucitas, de manera sencilla, es que este conflicto no se soluciona recurriendo a acciones de protesta social, sino que la solución se alcanza recurriendo a los espacios institucionales adecuados (los juzgados), donde son actores individuales con pericia técnica los que pueden participar, marginando de la escena a los actores colectivos (movimientos sociales).

Lo anterior se observa al constatar el peso que tienen las referencias al proceso contencioso administrativo seguido para determinar la legalidad o ilegalidad de la concesión minera otorgada a Industrias Infinito, en contraste con la poca referencia presente en el contenido de los medios a las 
actividades de protesta realizada contra la minería (ver Cuadro 3 y 4). Si bien es cierto que no se puede negar la importancia del proceso judicial como mecanismo utilizado para buscar dirimir este conflicto, al mismo tiempo que fue una estrategia promovida por los propios grupos ambientalistas, no fue la única acción realizada por estos grupos con ese propósito; por lo tanto, puede pensarse que hay una omisión consciente de los medios de reportar información relacionada con las acciones de protesta, con el propósito de transmitir a la población la idea que los canales institucionales son los adecuados para que la población posicione sus demandas y no recurra a la protesta social para ello.

Esto se puede también percibir en el hecho que los medios de prensa estudiados no dan ningún tipo de seguimiento a las acciones de protesta social que se realizan en la comunidad de Crucitas y alrededores, y solo a las que se realizan (o vinculan) en la ciudad capital del país: San José; esto a pesar que medios e información extranjera (Bravo, La Prensa: 17/10/ 2010) y en canales alternativos de comunicación, ${ }^{5}$ se realizan distintos reportajes sobre las acciones realizadas por personas de la comunidad de Crucitas con el propósito de detener el desarrollo de la minería a cielo abierto en la zona

Siguiendo lo anterior, no es de extrañar entonces que al identificar los distintos actores presentes en el contenido de los medios estudiados, los grupos o movimientos ambientalistas sean retratados como algo difuso, mientras ganan notoriedad los especialistas (por ejemplo, el geólogo Allan Astorga y el abogado ambientalista Álvaro Sagot), personas que tienen el manejo de los conocimientos necesarios para presentar argumentos en contra de Crucitas en el espacio técnico-jurídico en el que se desarrolla la discusión final sobre la realización de este proyecto.

Parece entonces que el contenido de los medios analizados apunta a que, en el caso de Crucitas, nos encontramos en una individualización de la protesta social, donde los actores colectivos pierden su supremacía como principales promotores y gestores de la protesta social, y son excluidos de un espacio -el jurídico- donde solo los actores individuales con determinadas capacidades pueden incidir y llevar a cabo las acciones de oposición.

Por último, a pesar que en el contenido de los medios de información se presenta que se resuelve el conflicto inmediato, el desarrollo del proyecto minero por parte de Industrias Infinito en Crucitas, no se aborda la discusión política del problema de fondo sobre el modelo de desarrollo que debe seguir Costa Rica, así como su relación con la naturaleza, el cual no se soluciona. Si bien en el contenido de los medios se hace referencia a intentos por parte de actores políticos, como la Asamblea Legislativa, de generar acciones para prohibir la actividad minera en el país, el problema mayor en el que se enmarca no se discute, ni tampoco se le permite a la sociedad tener un espacio de participación en la generación de propuestas y búsqueda de soluciones respecto a este, siendo los espacios formales de toma de decisiones políticas los únicos que pueden participar en este proceso. 
Polis, Revista Latinoamericana, Volumen 12, $N^{\circ}$ 36, 2013

\section{Notas}

${ }^{1}$ El presente texto muestra algunas conclusiones de un proyecto de investigación mayor, titulado Análisis político del discurso informativo de la prensa escrita sobre movimientos sociales en Costa Rica; realizado conjuntamente en el 2011 por el Programa Umbral Político del Instituto de Estudios Sociales en Población (IDESPO) de la Universidad Nacional (UNA) y la Escuela de Ciencias Políticas de la Universidad de Costa Rica (UCR). El autor quiere dejar constancia de su agradecimiento a los miembros del Programa Umbral Político y, en especial, a la Licenciada. Sindy Mora Solano, por sus valiosos comentarios y observaciones realizadas sobre el presente trabajo, no obstante, cualquier omisión u error presente en el mismo es de responsabilidad exclusiva del autor.

${ }^{2}$ A efectos de simplicidad o brevedad explicativa, en adelante se denominará así el proyecto y las situaciones conexas

${ }^{3}$ Para facilitar el procesamiento de la información y la identificación de palabras claves en el contenido de las noticias, se utilizó el programa informático Wordsmith, el cual es un programa informático que permite procesar grandes cantidades de texto con el propósito de obtener listas de palabras (listado de las palabras que aparecen con mayor frecuencia dentro del texto), así como identificar palabras clave (observar en que frases es utilizada una determinada palabra)

${ }^{4}$ La Nación se publica de manera diaria (lunes a domingo), Diario Extra y El País se publican de lunes a sábado y el Semanario Universidad se publica una vez a la semana

${ }^{5}$ Un ejemplo es el video reportaje Abriendo caminos a Crucitas, dirigido por Jeffery López; en el cual se muestran diferentes argumentos y acciones realizadas por los grupos comunales opositores a la minería en Crucitas; y el cual se dio a conocer en Youtube: http:// www.youtube.com/watch?v=Hn9muHxnGlM 


\section{Bibliografía}

Bustos Mora, G. (2011), “La mirada mediática hacia Tierra Dominicana: una aproximación desde el Análisis Crítico del Discurso (ACD)” [108124] Revista Rupturas No 1 Vol 1. Disponible en: http://investiga.uned.ac.cr/ rupturas/images/articulos/vol1/num1/mirmed.pdf

Casimigilia, G. y Tusón, A. (1999), Las cosas del decir. Editorial Ariel, Barcelona.

De Zan, J. (2006) “Los sujetos de la política. Ciudadanía y Sociedad Civil”. Tópicos, $\mathrm{N}^{\mathrm{o}} 14$.

Esteinou Madrid, F. J. (1992), Los medios de comunicación y la construcción de la hegemonía. Trillas, México DF.

Krippendorff, K. (1997), Metodología de análisis de contenido. Teoría y prática. Paidos Ibérica S.A., Barcelona.

Luhmann, N. (2007), La realidad de los medios de masas. Anthropos Editorial, Barcelona.

McQuail, D. (1996), Introducción a la teoría de la comunicación de masas. $2^{\text {a }}$ Ed. Rev. Paidós Comunicación, México DF.

Meso Ayerdri, K. (2008), “Tipología de los cibermedios vascos y modelos de producto. Estado de la cuestión sobre el estudio del fenómeno del ciberperiodismo desde la UPV-EHU”. En López García, G. (Ed.) Comunicación local y nuevos formatos periodísticos en internet: cibermedios, confidenciales $y$ weblogs. Servei de publicacions de la Univesitat de València, Valencia. Disponible en: http://cibermediosvalencianos.es/ ComunicacionLocal.pdf

Mora S., S. y García, A. (2008), “La representación de los actores sociales en el referéndum en la prensa escrita: La Nación y Diario Extra” [89-105] Revista de Ciencias Sociales $\mathrm{N}^{\circ} 121$.

Murillo Medrano, J. y Vergara Heidke, A. (2004), “Una propuesta de análisis textual a partir de los postulados del análisis crítico del discurso”. Revista de Filología y Lingüística de la Universidad de Costa Rica, XXX(1).

Shoemaker, P. J. y Reese, S. D. (1994), La mediatización del mensaje. Teorías de las influencias en el contenido de los medios de comunicación. $1^{\mathrm{a}}$ Edición. Editorial Diana, México D.F.

van Dijk, T. A. (2000), “El discurso como interacción de la sociedad.” En: van Dijk, Teun A. (Comp.) El discurso como interacción social. Estudios sobre el discurso II: Una introducción multidisciplinaria. Editorial Gedisa, Barcelona. 
Polis, Revista Latinoamericana, Volumen 12, $N^{\circ}$ 36, 2013

\section{Artículos de periódicos}

Artavia, B., “Ambientalistas quieren juicio para desnudar los informes técnicos”. Diario Extra, 15/6/2010.

Ídem, "La Municipalidad de San Carlos pide anular permisos de Crucitas". Diario Extra, 29/5/2010.

Ídem, “El 77\% de los Ticos se oponen a la minería a cielo abierto”. Diario Extra, 2/7/2010.

Ídem, “Sala IV rechaza revisar voto de Crucitas”. Diario Extra, 14/5/2010.

Bravo, J., “Mantienen protesta contra Las Crucitas”. La Prensa (Nicaragua), 17/10/2010 En: http://www.laprensa.com.ni/2010/10/17/nacionales/ 40925.

Chacón, V., "Resoluciones de tribunales en el 2010 desnudaron yerros del gobierno de Arias”. Semanario Universidad, 7/12/2010.

Cordero G., S., “Minera interpondrá demandas contra ambientalistas”. Diario Extra, 17/11/2010.

Ídem, “Será ilegal tramitar proyectos de minería en el país”. Diario Extra, 11/2/2011.

Ídem, “Crucitas no cumplió con requisitos en materia química”. Diario Extra, 12/10/2010.

Ídem, “Hoy se llevará a cabo audiencia de Crucitas”. Diario Extra, 11/08/ 2010.

Ídem, “Tribunal da curso a juicio contra Crucitas”. Diario Extra, 20/09/ 2010.

Ídem, “Nueva demanda contra Crucitas no detiene proceso". Diario Extra, 15/09/2010.

Ídem, “Listos para Romería por Crucitas”. Diario Extra, 12/07/2010

Ídem, “¡Ya les falta menos para llegar a Crucitas!”. Diario Extra, 16/07/ 2010 .

Ídem, “Caminante contra Crucitas de nuevo a Casa Presidencial”. Diario Extra, 19/07/2010.

Ídem, “Cuenta regresiva a futuro de Crucitas”. Diario Extra, 24/11/2010.

Córdoba. J., “Minera gasta \$149 millones en publicidad: Empresa de 
exasesor de Arias realiza publicidad de Minera Crucitas”. Semanario Universidad, 30/6/2010.

Ídem, “Abogados critican papel de la Procuraduría en caso de Crucitas”. Semanario Universidad, 25/1/2011.

Ídem, “Acusan a Infinito Gold por campaña contra grupos de oposición”. Semanario Universidad, 16/11/2010.

Ídem, "Decreto de moratoria deja puerta abierta para minería de otros metales”. Semanario Universidad, 2/6/2010.

Ídem, "La SETENA quedó al descubierto con sentencia sobre Crucitas". Semanario Universidad, 12/1/2011.

Ídem, "Procurador que apeló sentencia sobre Crucitas tendría conflicto de interés”. Semanario Universidad, 23/2/2011.

Ídem, Alfio Piva: "No queremos una Costa Rica llena de huecos para sacar oro”. Semanario Universidad, 16/6/2010.

Ídem, “Tribunal dio la razón a ambientalistas y académicos en juicio sobre mina en Las Crucitas”. Semanario Universidad, 1/12/2010.

Ídem, "Grupo Llamado Urgente: Especialistas critican resolución de la Sala sobre Crucitas”. Semanario Universidad, 30/7/2010.

CRH/EFE (2009, abril 16) "Sala Constitucional rechaza recurso contra minería en Crucitas”. Costa Rica Hoy. En: http://costaricahoy.info/nacionales/sala-constitucional-rechaza-recurso-contra-mineria-en-cruicitas/ 50126/

EFE, “Arias pide seguridad jurídica y dice que no teme a proceso por caso de mina”. El País, 26/11/2010.

Elpais.cr., “Abogados ambientalistas celebran fallo contra minera de oro a cielo abierto en Costa Rica”. El País, 25/11/2010.

Ídem, “Ambientalistas inician marcha contra mina en Costa Rica”. El País, 12/07/2010.

Ídem, “Arias sobre Juicio Crucitas: 'El que nada debe nada teme’ ”. El País, 26/11/2010.

Ídem, "Chinchilla continúa política ambiental de los Arias en el SENARA”. El País, 27/5/2010.

Ídem, “Chinchilla no dará más permisos para minería a cielo abierto pero no habla de Crucitas”. El País, 14/07/2010. 
Polis, Revista Latinoamericana, Volumen 12, $N^{\circ}$ 36, 2013

Ídem, "Costa Rica dice que proyecto minero no contradice política verde”. El País, 29/07/2010.

Ídem, “Defensa de minera Crucitas se derrumba con prueba aportada por ambientalistas”. El País, 23/10/2010.

Ídem, “Detectan más irregularidades en permiso para manejo de tóxicos en Crucitas”. El País, 13/05/2010.

Ídem, "Europa prohíbe el cianuro y en Costa Rica es de conveniencia nacional”. El País, 14/05/2010.

Ídem, "Extranjeros orientales destruyen almendro amarillo en Cutris de San Carlos”. El País, 11/07/2010.

Ídem, “Fundación Arias reconoce oferta de socio de proyecto minero”. El País, 28/04/2011.

Ídem, “Gobierno dejará en manos de tribunales caso de mina, pese a huelga de hambre”. El País, 15/10/2010.

Ídem, "Infinito Gold rehuye debate sobre minería a cielo abierto en la U. Latina”. El País, 23/06/2010.

Ídem, “Juicio Crucitas: 'Desviación de poder' del Gobierno de Arias, señalaron jueces”. El País, 15/12/2010.

Ídem, “Juicio Crucitas: Ambientalista reta a PGR a explicar desaparición camino público”. El País, 13/11/2010.

Ídem, "Las graves ilegalidades de los permisos para explotar oro en Crucitas”. El País, 19/12/2010.

Ídem, “Laura Chinchilla anunció que Gobierno no anulará el proyecto Crucitas”. El País, 27/7/2010.

Ídem, "Minera Crucitas niega haber donado dinero a Fundación de ex presidente Arias”. El País, 29/04/2011.

Ídem, “Minera de Crucitas reconoce la «apertura» de Oscar Arias a favor del proyecto”. El País, 25/09/2010.

Ídem, "Minera promociona «bondades» con el ambiente en gran campaña millonaria”. El País, 3/06/2010.

Ídem, "Minería a cielo abierto de Crucitas en manos de Laura Chinchilla”. El País, 23/07/2010.

Ídem, “Minería, arbitraje y amenazas contra Costa Rica por caso Crucitas”. 
El País, 3/08/2010.

Ídem, "Minería, seguridad y política social, primeros decretos de Chinchilla Crucitas queda por fuera del decreto minero de la nueva presidenta”. El País, 9/05/2010.

Ídem, “Organizan protestas frente a Procuraduría en Costa Rica contra proyecto minero”. El País, 31/10/2010.

Ídem, “Oscar Arias, cuatro años de confrontación social”. El País, 8/05/ 2010.

Ídem, “Procuradora rendirá cuentas a diputados por caso Crucitas”. El País, 21/01/2011.

Ídem, "Procuradora responderá cuestionamientos sobre Crucitas en una semana”. El País, 28/01/2011.

Ídem, "Respaldo del Gobierno de Costa Rica a minera Crucitas es imprudente, dice PAC”. El País, 28//07/2010.

Ídem, "Solicitan a Sala IV revisar voto sobre minería de oro a cielo abierto en Costa Rica”. El País, 11/05/2010.

Ídem, "Viceministro y asesor de Laura Chinchilla ha sido un aliado de Crucitas”. El País, 30/07/2010.

Ídem, "Juicio contra minería en Costa Rica fortalece rumbo favorable al ambiente”. El País, 2/11/2010.

Ídem, "Tribunal Contencioso Administrativo da curso a juicio contra minera de Crucitas”. El País, 17/09/2010.

Ídem, “Inicia juicio que decidirá futuro de polémica mina de oro en Costa Rica”. El País, 4/10/2010.

Ídem, “Juicio sobre minería a cielo abierto en Costa Rica se podrá prolongar”. El País, 5/10/2010.

Ídem, “Juicio contra minera en Costa Rica en maratónica sesión este viernes”. El País, 9/10/2010.

Ídem, "En juicio contra minería en Crucitas hoy debaten nueva prueba de ambientalistas”. El País, 29/10/2010.

Ídem, “Concentración y concierto hoy contra mina Crucitas en Día Mundial del Ambiente”. El País, 5/06/2010.

Ídem, “Marcha contra minería en Crucitas saldrá el próximo lunes de Casa 
Polis, Revista Latinoamericana, Volumen 12, $N^{\circ}$ 36, 2013

Presidencial”. El País, 5/07/2010.

Ídem, “Activistas protestan contra minería con caminata de 170 kilómetros”. El País, 12/07/2010.

Ídem, "Caminata contra minería en Crucitas recibirá refuerzos este jueves”. El País, 15/07/2010.

Ídem, “Activistas harán huelga de hambre contra proyecto minero en Crucitas”. El País, 1/10/2010.

Ídem, “Ayuno frente a Casa Presidencial para rechazar minería en Crucitas”. El País, 2/10/2010.

Ídem, “Ayuno entra en segundo día, con apoyo popular e intimidación policial”. El País, 10/10/2010.

Ídem, "La Nación manipula información para criminalizar protesta social”. El País, 12/05/2010.

Esquivel, J., “Rodrigo Arias criticó fallo sobre Crucitas”. La Nación, 25/ 11/2010.

Ídem y Agüero, M., “Rodrigo Arias fustiga anulación de plan minero”. $L a$ Nación, 25/11/2010.

Fuentes, F. "Huelguista contra mina de Crucitas fue hospitalizada”. La Nación, 26/10/2010.

Loaiza, V., “Agrupación antiminera protestó en Zapote”. La Nación, 16/ 08/2010.

Ídem, “Extensas declaraciones alargan juicio Crucitas”. La Nación, 12/10/ 2010.

Ídem y Murillo, A., “Gobierno minimiza huelga de hambre por Crucitas”. La Nación, 19/10/2010.

Mata, E., “Ejecutivo pone énfasis en plan contra la minería”. La Nación, 2/ $8 / 2010$

Murillo, A., “Chinchilla estudia decreto sobre minería en Crucitas”. La Nación, 15/7/2010.

Ídem, “Chinchilla toma más días para definir sobre Crucitas”. La Nación, 23/7/2010.

Ídem, “Gobierno calcula \$1.700 millones como indemnización por Crucitas”. La Nación, 26/7/2010. 
Nuñez, M., "Harán caminata de San Carlos a San José: Lucha contra minería en Crucitas toma fuerza”. Semanario Universidad, 25/08/2010.

Oviedo, E., “Gobierno descarta anular concesión minera de Crucitas”, $L a$ Nación, 27/7/2010.

Ramírez, E., "Paz con la Naturaleza propuso decreto: Gobierno desechó moratoria más fuerte para minería”. Semanario Universidad, 23/6/2010.

Ídem, “Piden a Sala IV rectificar fallo sobre minería en Crucitas”. Semanario Universidad, 12/5/2010.

Salazar, "Crucitas: A dos años de la firma de un decreto inconveniente y maloliente”. El País, 13/10/2010.

Ídem, “Transnacionales sobornan a técnicos, a políticos y a otros”. El País, 10/05/2010.

Vizcaíno, I., “Juicio decidirá sobre legalidad de permisos dados a Crucitas”. La Nación, 4/10/2010.

Ídem y otros, “Tribunal anula concesión de mina Crucitas”. La Nación, 24/ 11/2010.

Recibido: 22.06.2012

Aceptado: 10.04.2013 